Gefässchirurgie 2008 · 13:51-54 DOI 10.1007/s00772-007-0564-6

Online publiziert: 13. Dezember 2007

(c) Springer Medizin Verlag 2007

\author{
S. Deyle ${ }^{1,4} \cdot$ A. Leiser ${ }^{1} \cdot$ M. Binkert ${ }^{2} \cdot$ F. Bannwart $^{3} \cdot$ B. Lerf ${ }^{1}$ \\ ${ }^{1}$ Klinik für Chirurgie, Kantonsspital Zug \\ ${ }^{2}$ Gefässpraxis Zug \\ ${ }^{3}$ Institut für Pathologie, Stadtspital Triemli, Zürich \\ ${ }^{4}$ Klinik für Viszerale und Transplantationschirurgie, Inselspital Bern
}

\title{
Zystische Adventitiadegeneration (CAD) als Ursache einer invalidi- sierenden Claudicatio intermittens
}

dorsalen Zugang zeigte sich ein langstreckiges wandverdicktes, zystisch aufgetriebenes erkranktes Poplitealsegment. Aus einer größeren Zyste entleerte sich eine gallertige, zähflüssige, gelbliche Flüssigkeit (- Abb.3). Aufgrund der langstreckigen Veränderung von $35 \mathrm{~mm}$ erfolgte die Segmentresektion der A. poplitea mit anschließender Rekonstruktion mittels eines autologen Veneninterponats (reversed V. saphena parva). Die histologische Untersuchung ergab Zysten in der Adventitia mit Zonen mukoider Degeneration (• Abb. 4). Postoperativ wurde der Patient oral antikoaguliert (Phenprocoumon) mit einer Ziel-INR zwischen 2 und 3. Der stationäre Verlauf sowie die Verlaufskontrollen nach ein und zwei Jahren gestalteten sich komplikationslos mit kräftig palpablen Fußpulsen. Duplexsonographisch zeigten sich ebenfalls einwandfreie Verhältnisse am Interponat.

\section{Diskussion}

Die zystische Adventitiadegeneration (CAD) ist ein eher seltenes gefäßchirurgisches Krankheitsbild und wurde erstmals 1947 von Atkins [1] im Bereich der A. iliaca externa beschrieben. 1954 folgte von Ejrup [3] eine Publikation über die CAD im Bereich der A. poplitea. Die bis heute noch gültige Bezeichnung wurde 1957 von Hiertonn u. Lindberg geprägt. In der Literatur sind bis heute weit über 350 Fälle dokumentiert, und es wird eine Inzidenz von 1:110o der Patienten mit Claudicatiosymptomatik angegeben. In etwa $80 \%$ der Fälle sind Männer betrof- fen mit einem Durchschnittsalter von ca. 40-50 Jahren [4]. Typischerweise fehlen meist die üblichen vaskulären Risikofaktoren und bekannten Zeichen der generalisierten arteriellen Verschlusskrankheit. Die betroffenen Patienten haben eine normale Lebenserwartung, da in der Regel keine systemische Arteriosklerose vorliegt. Hauptlokalisation ist die A. poplitea, es können aber auch andere periphere Arterienabschnitte betroffen sein [8]. Selbst über eine Beteiligung der Venen wurde bereits berichtet [9].

Gerade bei eher jungen Patienten mit sehr wechselhaften Ischämiebeschwerden ohne Arterioskleroserisiko muss differenzialdiagnostisch neben dem poplitealen Entrapmentsyndrom auch die zystische Adventitiadegeneration in Betracht gezogen werden. Aufgrund von adventitieller Zystenbildung gelenknaher Arterien führt die Erkrankung zu einer Kompression des Gefäßlumens mit Verschlusssymptomatik. Somit ist eine fluktuierende Symptomatik von beschwerdefreien Intervallen bis hin zu einer deutlichen Begrenzung der Gehstrecke auf wenige Meter nicht ungewöhnlich [11] und ist möglicherweise auf einen wechselnden Füllstatus der Zyste bei einem Verbindungsgang zum Gelenk zurückzuführen. Die zystischen Strukturen in der Adventitia können ein- oder mehrkammerig sein, solitär oder multipel auftreten [4]. Wegweisend für die Diagnose einer CAD sind die Gelenknähe der Läsion, das Alter der Patienten sowie der plötzliche Beginn der Beschwerden ohne sicheres unmittelbar vorausgehendes Trauma. Die Ätiologie
Der Patient wurde einem gefäßchirurgischem Eingriff zugeführt. Über einen 


\section{Der interessante Fall}

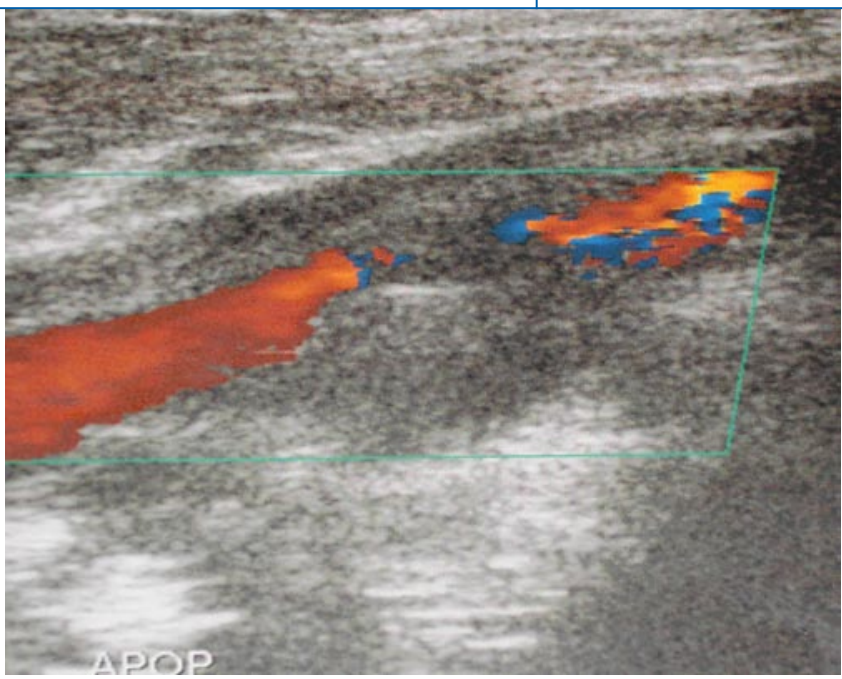

Abb. $1<$ Duplexsonographischer Längsschnitt mit echoarmer Raumforderung und exzentrischer Stenose der A. poplitea mit homogenem Zysteninhalt

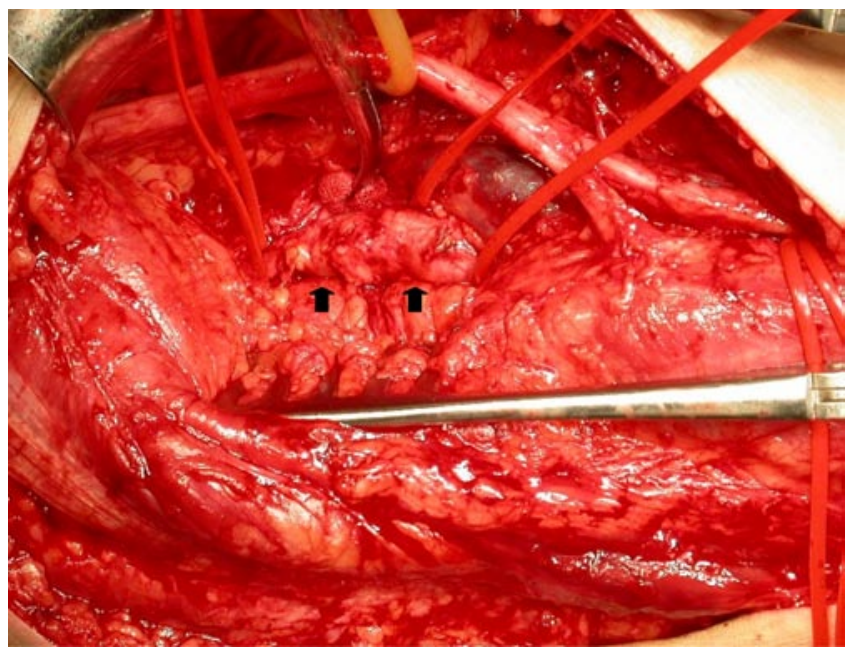

Abb. $3<$ Intraoperativer Situs nach Eröffnen der Adventitiazyste

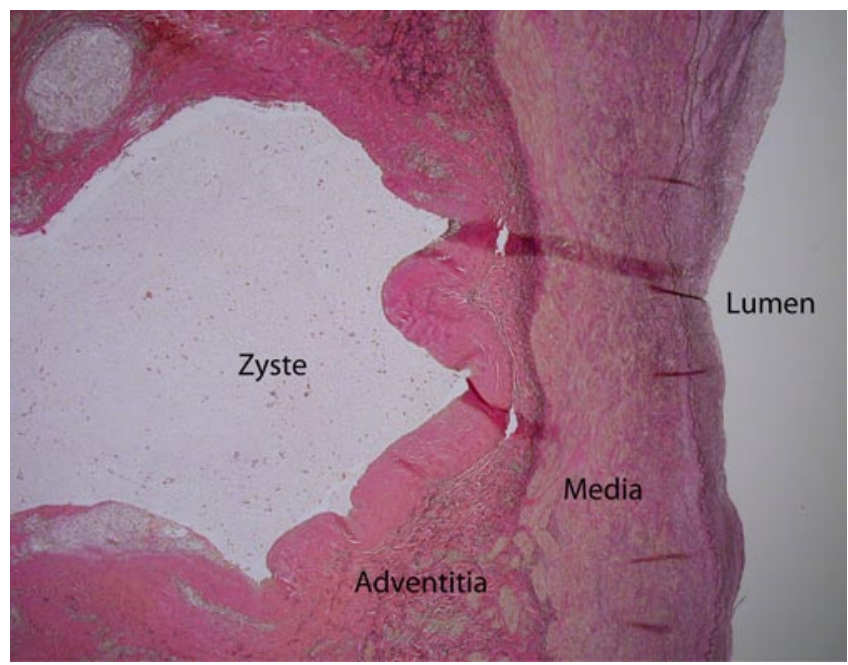

Abb. $4<$ Histologie des Operationspräparates mit mukoidem Material

ist nach wie vor nicht vollständig geklärt; verschiedene Mechanismen werden diskutiert.

Zum einen kann aufgrund der Gelenknähe eine durch repetitive Mikrotraumen hervorgerufene adventitielle mukoide De-

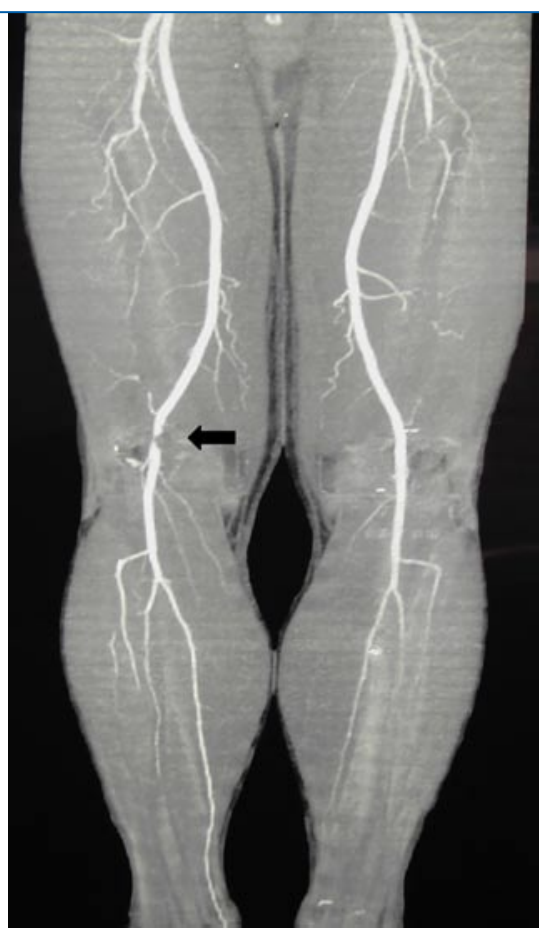

Abb. $2 \triangle$ Angiographische Darstellung der hochgradigen uhrglasförmigen Stenose der A. poplitea

fach beschrieben $[7,8]$. Gemäß einer weiteren Hypothese wird eine Herniation von Synovialgewebe in die Adventitia postuliert [8].

Zur Objektivierung des stenosierenden Gefäßprozesses ist die diagnostische Maßnahme der ersten Wahl zum einen die Duplexsonographie. Hierbei werden die Einengung des arteriellen Lumens sowie die Zystenwand mit dem echoarmen, meist homogenen Inhalt dargestellt. Zum anderen basiert die klinische Diagnostik auf der charakteristischen angiographischen Erscheinung der Läsion in Form einer glatten, bogenförmigen oder uhrglasförmigen Stenose bei einem ansonsten unauffälligen Gefäßsystem, dem Scimitar-Zeichen. MRI/CTUntersuchungen geben zusätzliche Hinweise im Hinblick auf Ausmaß und Kammerung des zystischen Prozesses [11], stellen die angrenzenden Weichteil- und Knochenstrukturen genau dar und können zur Abgrenzung des Entrapmentsyndroms sinnvoll sein. Erschwert zeigt sich die Diagnostik bei geringem Füllungszustand der Zysten.

Obwohl es Beschreibungen einer spontanen, kompletten Rückbildung der Erkrankung gibt [10], wird eine invasive 
Therapie aufgrund von Persistenz oder Progredienz der Symptomatik in der Mehrzahl der Fälle notwendig. In den letzten Jahrzehnten wurden verschiedene Therapiekonzepte beschrieben. Von perkutanen, sonographisch gesteuerten oder offenen Zystaspirationen wurde mehrfach berichtet, wobei gerade das sonographisch gesteuerte Verfahren ohne Strahlenbelastung einfach und wenig zeitintensiv durchführbar ist [2]. Dieses Vorgehen ist jedoch im Langzeitverlauf insgesamt mit einer hohen Rezidivgefahr verbunden [11]. Aufgrund der Muzinviskosität und ggf. bestehenden Kammerung der Zyste gelingt eine Evakuation unter Umständen nicht vollständig. Klassisch chirurgisch besteht die Option eines gefäßerhaltenden oder -resezierenden Verfahrens. Bei intakter Media der Gefäßwand ohne Vorliegen eines arteriellen Verschlusses kann eine Resektion der Zyste oder eine partielle Wandresektion, ggf. mit Einbringung einer Patchplastik, vorgenommen werden [7]. Jedoch zeigt die alleinige Zystektomie ohne zusätzliche Resektion der angrenzenden betroffenen Adventitia ein hohes Rezidivrisiko. Gerade bei jungen Patienten sollte die gefäßerhaltende Therapie mittels „Exarterektomie“ evaluiert werden [12]. In Spätstadien mit arteriellem Verschluss, bei mehrkammerigen Zysten oder nach PTA-bedingter Zystenruptur ist die Implantation eines autologen Veneninterponats erforderlich $[4,5,6]$. Eine bestehende Stielverbindung zum Gelenk muss identifiziert und ligiert werden.

In unserem Fall haben wir uns aufgrund der langstreckigen Veränderung bei persistierendem Stadium IIb und unter Berücksichtigung des bestehenden Leidensdruckes des beruflich körperlich sehr aktiven Patienten zur operativen Sanierung entschlossen. Die Läsion wurde reseziert und durch ein autologes Veneninterponat ersetzt. Der dorsale $\mathrm{Zu}$ gang, wie wir ihn bei unserem Patienten gewählt haben, ermöglicht eine gute Kontrolle über das betroffene popliteale Segment. Die V. saphena parva bietet sich bei diesem Zugang direkt als Interponat an, sofern das Lumen weit genug ist. Bei fehlender Arteriosklerose ist mit einem Veneninterponat ein gutes Langzeitergebnis $\mathrm{zu}$ erwarten [6].

\section{Gefässchirurgie 2008 · 13:51-54 DOI 10.1007/s00772-007-0564-6}

(c) Springer Medizin Verlag 2007

\section{S. Deyle · A. Leiser · M. Binkert · F. Bannwart · B. Lerf Zystische Adventitiadegeneration (CAD) als Ursache einer invalidisierenden Claudicatio intermittens}

\section{Zusammenfassung}

Bei einem 45-jährigen Patienten mit wechselhaften Ischämiebeschwerden und initial limitierter Gehstrecke rechts von höchstens $50 \mathrm{~m}$ führten die duplexsonographischen und angiographischen Abklärungen zur Verdachtsdiagnose einer zystischen Adventitiadegeneration. Intraoperativ zeigte sich ein zystisch aufgetriebenes Poplitealsegment mit Entleerung von gallertiger Flüssigkeit. Aufgrund der langstreckigen Veränderung erfolgte die Segmentresektion der A. poplitea und Rekonstruktion mittels eines autologen Veneninterponats. Die CAD ist ein seltenes Krankheits- bild, an das gerade bei jungen Patienten ohne systemische Arteriosklerose gedacht werden sollte. Die Therapie ist je nach Fall chirurgisch erhaltend durch Zystenresektion mittels „Exarterektomie" oder gefäßresezierend, insbesondere bei langstreckigen Veränderungen bzw. vollständiger Okklusion mittels Ersatz durch ein Veneninterponat.

\section{Schlüsselwörter}

Claudicatio intermittens - Poplitealstenose . Adventitiazyste · Duplexsonographie . Angiographie

\section{Cystic adventitial degeneration (CAD) as a cause of disabling intermittent claudication}

\section{Abstract}

Duplex sonography and angiography revealed cystic adventitial degeneration (CAD) in a 45-year-old patient with intermittent claudication of the right calf and an initial maximum walking distance of $50 \mathrm{~m}$. Intraoperatively we found a cystic lesion containing gelatinous fluid in the adventitial layer of the popliteal artery. Because of the extended lesion, resection of the involved segment was performed, followed by autologous venous reconstruction. CAD is a rare disease and should be considered in young patients without systemic atherosclerosis. The surgical treatment is, depending on the case, either nonresectional by circumferential removal of adventitial cysts or resectional followed by autologous reconstruction in extended lesions or total occlusion of the artery.

\section{Keywords}

Intermittent claudication - Popliteal artery stenosis · Intramural cyst · Duplex sonography · Angiography 


\section{Hier steht eine Anzeige.}

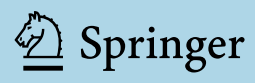

\section{Fazit für die Praxis}

Die zystische Adventitiadegeneration ist ein seltenes Krankheitsbild, welches jedoch gerade bei Männern mittleren Alters ohne systemische Arteriosklerose mit wechselhaften Ischämiebeschwerden in Betracht gezogen werden sollte. Die Diagnostik basiert auf der klinischen, sonographischen und - insbesondere bei vorgesehener gefäßchirurgischer Maßnahme - der angiographischen Untersuchung. Die Therapie der Wahl ist je nach Fall die chirurgische gefäßerhaltende Zystenresektion („Exarterektomie“) oder bei langstreckigem Befall bzw. arteriellem Verschluss der Ersatz des betroffenen Gefäßabschnittes durch ein Veneninterponat. Kathetertechnische interventionelle Verfahren kommen bei dieser Erkrankung ebenfalls zur Anwendung, jedoch besteht ein erhöhtes Rezidivrisiko.

\section{Korrespondenzadresse}

\section{Dr. S. Deyle}

Klinik für Viszerale und

Transplantationschirurgie,

Inselspital Bern,

3010 Bern, Schweiz

simone.deyle@insel.ch

Interessenkonflikt. Der korrespondierende Autor gibt an, dass kein Interessenkonflikt besteht.

\section{Literatur}

1. Atkins HJB, Key JA (1947) A case of myxomatous tumour arising in the adventitia of the left external iliac artery. Br J Surg 34: 426-427

2. Do DD, Braunschweig M, Baumgartner l et al. (1997) Adventitial cystic disease of the poplitea artery: Percutaneous US-guided aspiration. Radiology 203: 743-746

3. Ejrup B, Hierton T (1954) Intermittent claudication. Three cases treated by free vein graft. Acta Chir Scand 108: 217-230

4. Flanigan PD, Burnham SJ, Goodreau JJ, Bergan JJ (1974) Summary of cases of adventitial cystic disease of the popliteal artery. Ann Surg 189: 165175

5. Fox CJ, Rasmussen TE, O'Donnell SD (2004) Cystic adventitial disease of the popliteal artery. JVasc Surg 39: 1351

6. Hiertonn T, Karacagil S, Bergquist D (1995) Long term follow-up of autologous vein grafts. 40 years after reconstruction for cystic adventitial disease. Vasa 24: 250-252

7. Largiadèr J, Leu HJ (1984) Sogenannte zystische Adventitiadegeneration der Arteria poplitea mit Stielverbindung zum Kniegelenk. Vasa 13: 267271
8. Leu HJ, Largiader J, Odermatt B (1984) Pathogenesis of the so-called cystic adventitial degeneration of the peripheral blood vessels: Virchows Arch A Pathol Anat Histopathol 404: 289-300

9. Paty PS, Kaufmann Jl, Koslow AR et al. (1992) Adventitial cystic disease of the femoral vein: a case report and review of the literature. J Vasc Surg 15: 214-217

10. Pursell R, Torrie EP, Gibson M, Galland RB (2004) Spontaneous and permanent resolution of cystic adventitial disease of the popliteal artery. J R Soc Med 97: 77-78

11. Schaeberle W, Eisele R (1996) Die sonographische Diagnostik, Verlaufskontrolle und Therapie der zystischen Adventitiadegeneration. Ultraschall Med 17: 131-137

12. Stierli P, Mauch J, Koella C et al. (2005) Circumferential removal of the adventitia for cystic degeneration of the popliteal artery. Br J Surg 92: 56-57 